\title{
Spectral CT of the abdomen: Where are we now?
}

\author{
Sharon Z. Adam ${ }^{1,2^{*}}$ (D) Aviad Rabinowich ${ }^{1,2}$, Rivka Kessner ${ }^{1,2}$ and Arye Blachar ${ }^{1,2}$
}

\begin{abstract}
Spectral CT adds a new dimension to radiological evaluation, beyond assessment of anatomical abnormalities. Spectral data allows for detection of specific materials, improves image quality while at the same time reducing radiation doses and contrast media doses, and decreases the need for follow up evaluation of indeterminate lesions. We review the different acquisition techniques of spectral images, mainly dual-source, rapid kV switching and dual-layer detector, and discuss the main spectral results available. We also discuss the use of spectral imaging in abdominal pathologies, emphasizing the strengths and pitfalls of the technique and its main applications in general and in specific organs.
\end{abstract}

Keywords: Spectral CT, Dual energy CT, Abdominal Imaging, Mono-energetic images, lodine maps

\section{Key points}

- Spectral CT allows for material-specific analysis in addition to anatomical images of conventional CT.

- The main spectral acquisition techniques are dual source, rapid kV switching and dual layer detector.

- The most commonly used spectral applications are virtual non-contrast images, mono-energetic images at different keV levels and iodine concentration.

- Spectral data can be used to accentuate enhancement, reduce metal artifacts, decrease the number of scans in multiphasic imaging and characterize lesions.

\section{Introduction}

Spectral computed tomography (CT), also known as multi-energy $\mathrm{CT}$ or dual-energy $\mathrm{CT}$, was first hypothesized in the 1970s. However, it took years until the first multi-energy scanners were robust enough to be introduced into routine clinical practice in 2006, first as dual

\footnotetext{
*Correspondence: sharonad@tlvmc.gov.il

${ }^{1}$ Department of Diagnostic Radiology, Tel Aviv Sourasky Medical Center, 6 Weizmann St., 6423906 Tel Aviv, Israel

${ }^{2}$ Sackler School of Medicine, Tel Aviv University, Tel Aviv, Israel

Full list of author information is available at the end of the article
}

source scanners (utilizing two tubes with different voltages) and rapid kilo-voltage $(\mathrm{kV})$ switching scanners, and lately also as dual layer detector scanners.

Conventional CT provides anatomical and morphological evaluation of organs and tissues, with mostly qualitative assessment of tissues in relation to adjacent tissues, considering that the images are dependent on the energy level selected during acquisition. Spectral $C T$ adds a new dimension to the images acquired, with material decomposition supplying data regarding true composition of specific materials in the scanned tissues, allowing for accurate assessment of iodine concentration, subtraction of iodine from contrast-enhanced images, and also supplying better quality anatomical images using monoenergetic reconstructions.

We will discuss the principles and technical aspects of spectral CT acquisition, describe general advantages and disadvantages and also refer to organ specific applications in the abdomen.

\section{Spectral principles and technology}

Conventional CT images are generated from average attenuation values in multiple voxels which contain multiple tissues. These tissues may have overlapping attenuation at any given peak tube voltage $(\mathrm{kVp})$. The main contributors to attenuation coefficients are the 
photoelectric effect, which predominates in lower energy levels, is highly energy-dependent and related to high atomic numbers, and Compton scattering, which occurs at energies above 50 kilo-electron Volt $(\mathrm{keV})$, and is predominantly related to the electron density of a given material. The comparison of attenuation levels derived from two energy levels, high and low, can be utilized to calculate the photoelectric effect and Compton scattering. This allows for separation of tissues with similar attenuation in any single energy level. This is commonly referred to as material decomposition and is the basis for spectral CT imaging.

The datasets of the two energy levels can be acquired in multiple ways. The three main acquisition techniques currently in the market (Fig. 1) are-dual source acquisition (in which a low $\mathrm{kV}$ tube and a high $\mathrm{kV}$ tube scan simultaneously at an angle offset to achieve 2 energy spectra), rapid $\mathrm{kV}$ switching (in which the same tube rapidly switches from low to high $\mathrm{kV}$ constantly during its rotation) and detector-based spectral separation (in which the detector is a dual layer detector separating the energy levels after the beam has crossed the patient, detecting low energy photons in the top layer and high energy photons in the bottom layer), which is the newest technique, available commercially since 2016. Other techniques also exist, although not as commonly used, including "rotate-rotate" techniques (in which the tube alternates energy levels between rotations) and scanning using filter-based spectral beam splitting (split gold-tin filter resulting in spectral separation before the beam passes through the patient), which has also only recently been introduced into clinical practice [1].

Each technique and each manufacturer uses a different mathematical calculation to achieve spectral separation, thus resulting in different spectral separation accuracies [2], but all are considered robust and are in use clinically.
There are several main advantages and disadvantages to the different techniques. Detector-based spectral imaging has the advantage of acquiring spectral data for all scans without predetermining the need for spectral data ("always on"), with spectral data being able to be acquired retroactively, without changing scan protocols and available for all scan phases in multiphase studies. These scanners also provide true conventional CT images, unlike all other techniques which only provide the conventionalequivalent image which is a blended images of the two energy levels. Detector-based imaging also has the best temporal coherence because the different energies are all detected at the same time, and is affected the least by patient motion. Dual source spectral imaging has the advantage of two tubes, able to generate good image quality even in large patients, with the second tube able to be used in non-spectral mode to perform extremely fast scans, while also being the only technique limited by a small field-of-view for spectral data because of space limitations within the gantry, approximately $35 \mathrm{~cm}$ compared with $50 \mathrm{~cm}$ for other techniques. The main spectral acquisition techniques are able to provide scans with comparable radiation doses to single-energy CT, regardless of the technique [3-5]. A full comparison of technologies with their advantages and disadvantages is beyond the scope of this article and can be found in the literature $[1,6]$.

\section{General applications}

The main applications currently available for most spectral scanners are virtual non-contrast (VNC) images, mono-energetic images at different $\mathrm{keV}$ levels, iodine concentration, effective atomic number images, and other material density images, which are constantly being improved, with new applications also being developed. Some applications will be discussed separately here,
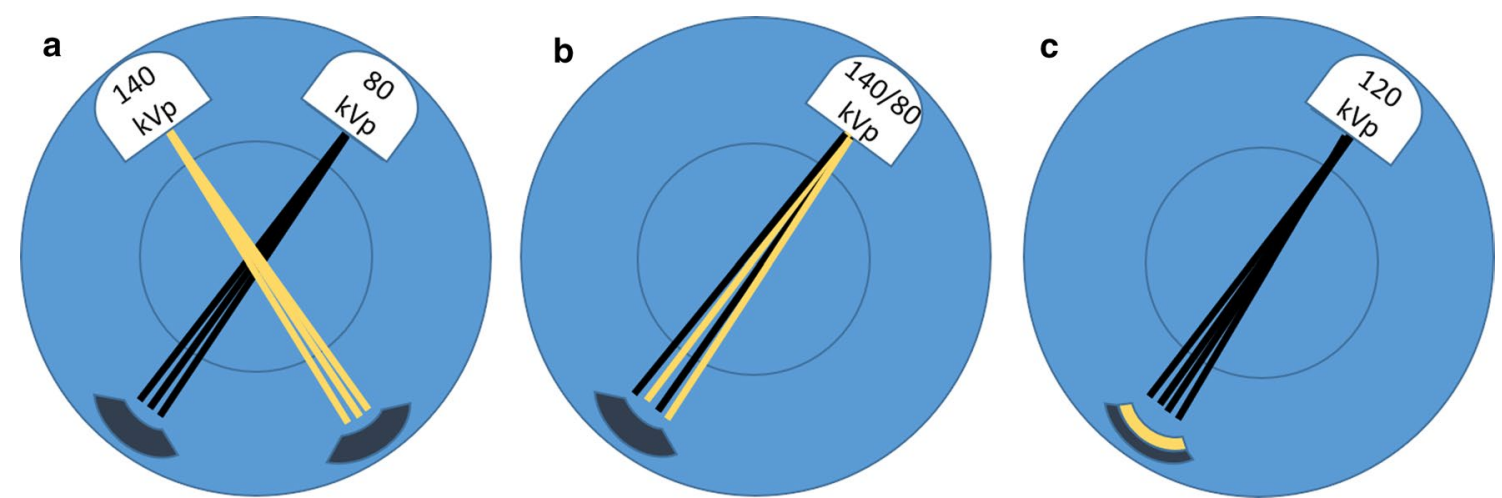

Fig. 1 The main acquisition techniques currently in the market for spectral imaging: dual source dual energy CT (a), rapid kV switching (b) and detector based spectral imaging (c) 
while others will be discussed in the organ-specific section later in the article.

\section{VNC images}

VNC images are created by subtracting all contrastenhanced structures from the acquired image. A robust VNC image will allow for reduction of the overall radiation exposure by decreasing the number of scans per protocol. This reduction can be substantial, ranging from 25 to 35\% [7], depending on site-specific protocols. VNC images are not affected by concentration or rate of injected contrast or by the phase they are acquired from (arterial vs. portovenous phase) [8].

However, replacing true unenhanced (true non-contrast, TNC) images by VNC images is only possible if we can use them in a similar fashion. Studies have shown that VNC images have comparable and adequate image quality $[7,9,10]$. It has also been shown that there is good correlation between attenuation on TNC images and VNC images [7, 9-11]. However, when one looks at the differences in attenuation numbers, quoted as under $15 \mathrm{HU}$ in $92.6-98.6 \%$ of measurements, with higher attenuation differences in fluid and fat $[7,9]$, one wonders as to the clinical applicability. TNC images in multiphasic examinations are used mainly for qualitative analysis as for the presence of calcifications and other hyperdense substances prior to contrast administration, assessment of pre-contrast attenuation in adrenal lesions or liver parenchyma, and quantitative analysis as part of enhancement measurements in a suspected lesion. As for evaluation of enhancement, one can argue that the inability to use the VNC as baseline for enhancement measurement is off-set by the data provided for iodine concentration. However, this is not the case for evaluation of calcifications, for instance in evaluation of arteries for calcified plaques and determination of severity of vessel occlusion. It is even more concerning in adrenal incidentalomas (Fig. 2), one of the most common lesions encountered in clinical practice. A possible difference of $15 \mathrm{HU}$, even when taking into account the positive bias in fat containing tissues (consistently higher attenuation in VNC compared with TNC) [9], may cause over diagnosis of adenomas as uncertain lesions and lead to unnecessary further imaging [10]. A more substantial body of knowledge is needed as for the correct threshold levels in such cases before the substitution can be made.

Therefore, it appears that VNC images are still not robust enough to replace TNC images in most clinical settings. We suggest such a substitution only in cases of borderline additional information gained by adding an unenhanced image. However, VNC images are a valuable tool in monophasic post-contrast protocols, in which previously many incidentalomas led to further imaging, which now can be avoided in some cases.

\section{Mono-energetic images}

The mono-energy application simulates a scan using a monochromatic $x$-ray beam and allows us to choose the energy level, in $\mathrm{keV}$, for the reconstruction. Available energy levels vary between spectral acquisition method

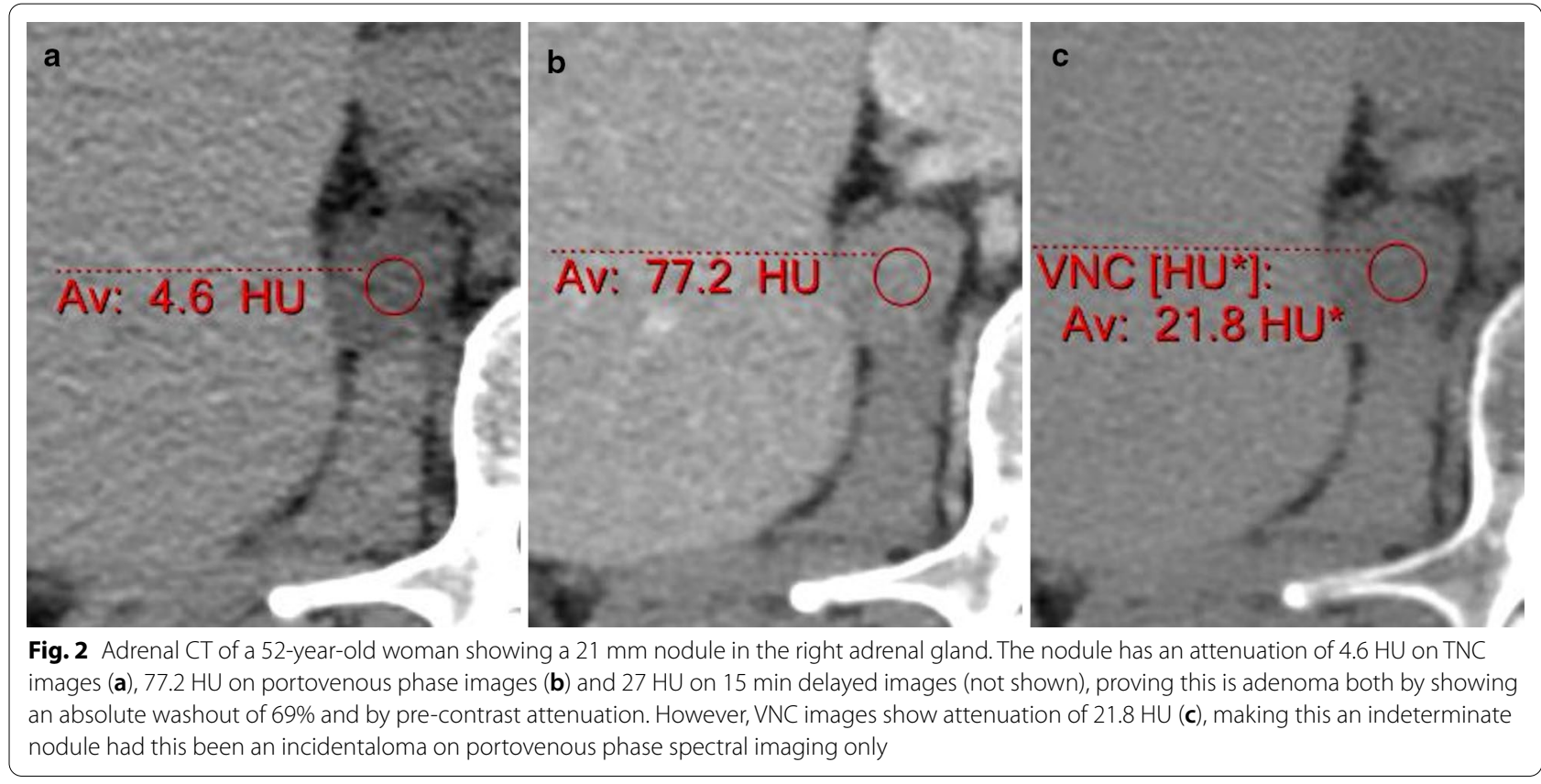


and manufacturer, ranging between 40 and $200 \mathrm{keV}$. The energy level equivalent to $120 \mathrm{kVp}$ is approximately $75 \mathrm{keV}$.

Conventional-equivalent mono-energetic images have been shown to have improved signal-to-noise ratio (SNR) and contrast-to-noise ratio (CNR) in contrast-enhanced scans, particularly in obese patients [12-14]. However, one must take into account the fact that measurements of HU cannot be used as with conventional images, and evaluating enhancement needs to be performed using iodine concentration images.

The attenuation of structures increases as the mean energy of the photons decreases, which is true for both non-spectral low $\mathrm{kVp}$ scanning and dual energy low $\mathrm{keV}$ mono-energetic settings. This is especially important for scans using contrast media, as a decrease in energy in the range approaching the K-edge of iodine (which is $33 \mathrm{keV}$ ) causes markedly increased attenuation of iodineenhanced structures [8].

Thus in spectral imaging, low $\mathrm{keV}$ mono-energetic images accentuate iodine-enhanced structures without increasing image noise and this allows us to decrease the amount of injected contrast media, which may be crucial in patients with renal impairment [8,15-23]. Studies have shown a feasible reduction of $70 \%$ in vascular studies and $50 \%$ in non-vascular studies, while considering that for optimal evaluation of hypervascular lesions the reduction should likely be more moderate, of approximately $35 \%$ [8]. However, the decision to decrease contrast doses is more complex, and needs to take into account overall injected volume and flow rate needed to achieve optimal enhancement. Also, as many institutions use multiple scanners only some of which are spectral scanners, this may entail individualized contrast protocols per scanner and per pathology (not only per body part), which may prove to be problematic for optimal workflow and may lead to more errors. Also one must consider that as the attenuation of injected contrast increases, the attenuation of positive oral contrast also increases [24, 25] which may cause more artifacts.

At the other end of the spectrum, high energy levels decrease beam-hardening artifacts and can be used for metal artifact reduction (Fig. 3) [26-28]. For both low $\mathrm{keV}$ and high $\mathrm{keV}$ reconstructions, one can choose the optimal energy level based on personal preference, and at our institution, using dual-layer spectral imaging, we prefer $50 \mathrm{keV}$ and $140 \mathrm{keV}$ images.

\section{lodine concentration images (iodine maps)}

This is a very significant application for lesion characterization. The generated images are equivalent to MRI subtraction images. Iodine maps give radiologists the confidence in evaluating contrast enhancement of a lesion, proving that some lesions do not show contrast material uptake and are benign, thus obviating the need for further evaluation. These images have been shown to have high accuracy regardless of acquisition parameters, as long as the radiation dose is within a clinical range [29, 30]. Although differences in accuracy between manufacturers (likely related to the difference in acquisition technique) have been reported for phantoms with errors in measurement questioning our ability to use reported threshold levels for certain pathologies [2, 31, 32], the error is under $10 \%$, which is an acceptable error, as long as correct thresholds are used. The correct threshold has not been adequately established yet. One recent study found liver cysts to have iodine uptake of $0.23 \pm 0.31 \mathrm{mg} /$ $\mathrm{mL}$, consistent with the accepted threshold of $0.5 \mathrm{mg} /$
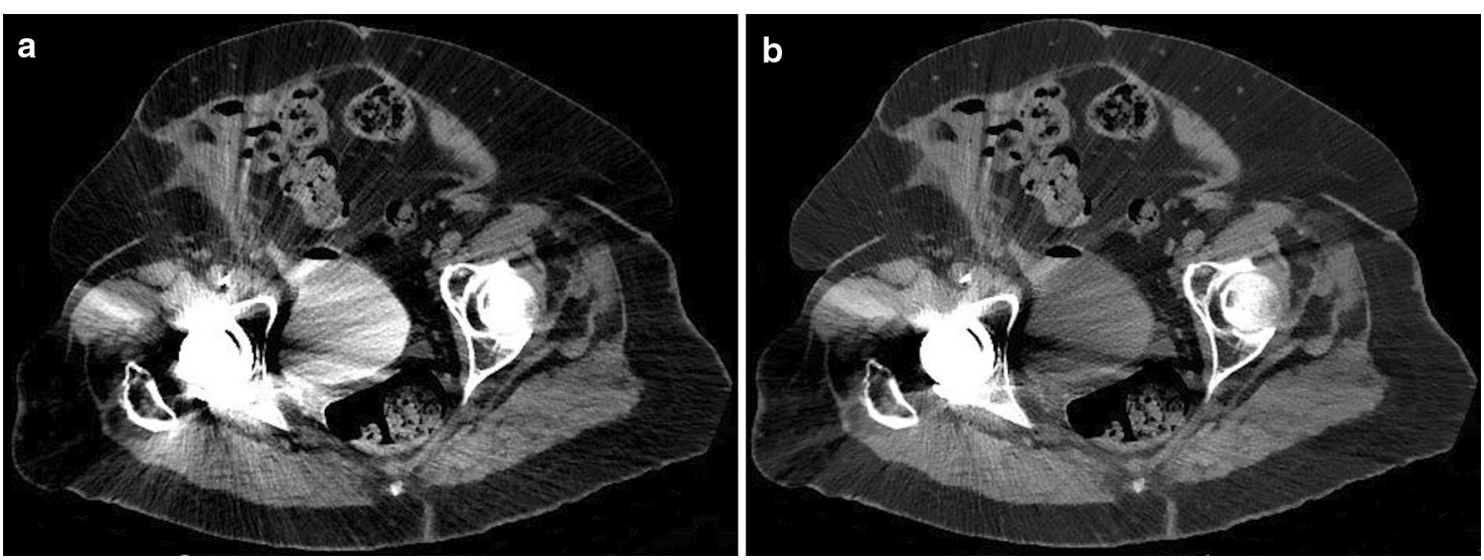

Fig. 3 CT cystography of a 70-year-old woman after renal transplantation with a prior total hip replacement. Beam hardening artifacts caused by metal hardware markedly degrade evaluation of pelvic structures (a). Spectral mono-energetic image at 140 keV shows improvement of artifacts, at a price of reduced attenuation of contrast in the bladder (b) 
$\mathrm{mL}$ across all scanners, but also found differences of up to $20 \%$ within the same lesion in different time points [33]. Another recent study [34] has found higher thresholds of $1.3 \mathrm{mg} / \mathrm{mL}$, and has suggested a more accurate approach of measuring iodine concentration by normalizing the results to the iodine concentration of the aorta, and using a normalized threshold of $0.3 \mathrm{mg} / \mathrm{mL}$. Until further data is accumulated, we believe radiologists should choose between a more conservative approach, using a lower threshold for enhancement and likely suggesting further imaging for lesions showing suspected mild iodine uptake, and a more liberal approach using a higher threshold which may lead to lesions being incorrectly diagnosed as non-enhancing. At our institution, where we use detector-based spectral CT, we choose the conservative approach. We also believe iodine concentration measurement on contrast-enhanced only studies, are not robust enough for omitting the unenhanced phase of multiphasic protocols (Fig. 4).

One of the most important uses for iodine maps in abdominal imaging is characterization of incidentally identified small lesions, the so called "too small to characterize" lesions. Although it is tempting to say iodine maps can be used to determine whether these small lesion are truly non-enhancing, one must remember that these images are still affected by the same partial volume artifacts which prevent us from reliably measuring the density (HU) of a lesion on conventional images. The true size threshold for determining the iodine content of a lesion using iodine maps is unknown.

\section{Organ specific applications}

Liver

The most intuitive use of spectral CT in the liver is for better visualization of hypervascular lesions using low $\mathrm{keV}$ mono-energetic images of the arterial phase (Fig. 5).
This applies to hypervascular tumors, such as hepatocellular carcinoma (HCC) and neuroendocrine tumors (NET), with studies showing better conspicuity, allowing for visualization of a larger number of lesions, with improved reader confidence [35-38]. However, the low $\mathrm{keV}$ images may reveal many small hypervascular lesions, some of which may be of uncertain significance, and therefore evaluation of such lesions should always be in the correct context, with concurrent evaluation of conventional images, in order to prevent over diagnosis. Using low $\mathrm{keV}$ images may also increase visualization of hypo-enhancing hepatic lesions, such as small hypovascular metastases, by increasing the attenuation of the surrounding liver [39].

Better visualization of blood vessels and extravasation on low $\mathrm{keV}$ images $[17,40]$ may lead to improved visualization of active hepatic hemorrhage in both traumatic and non-traumatic cases and may also lead to better evaluation of vasculature before and after liver transplantations.

Although iodine maps have been shown to be accurate in liver phantom models, their clinical use in evaluation of tumor response is still limited because of differences in the biological distribution causing inter-patient and intra-patient variability [33]. However, iodine quantification has been shown to improve accuracy of diagnosis of portal vein tumor thrombus, best evaluated using a threshold level of $0.9 \mathrm{mg} / \mathrm{mL}$ [41]. It may also potentially help to evaluate response to treatment in Budd-Chiari syndrome [42] and differentiate between liver metastases and abscesses [43]. Specifically for HCC, it has been shown that iodine concentration images improve the assessment of hypervascularity and washout [44]. It is also showing promise in differentiating between HCC and non-cancerous hypervascular cirrhotic nodules based on iodine content alone, regardless of washout,

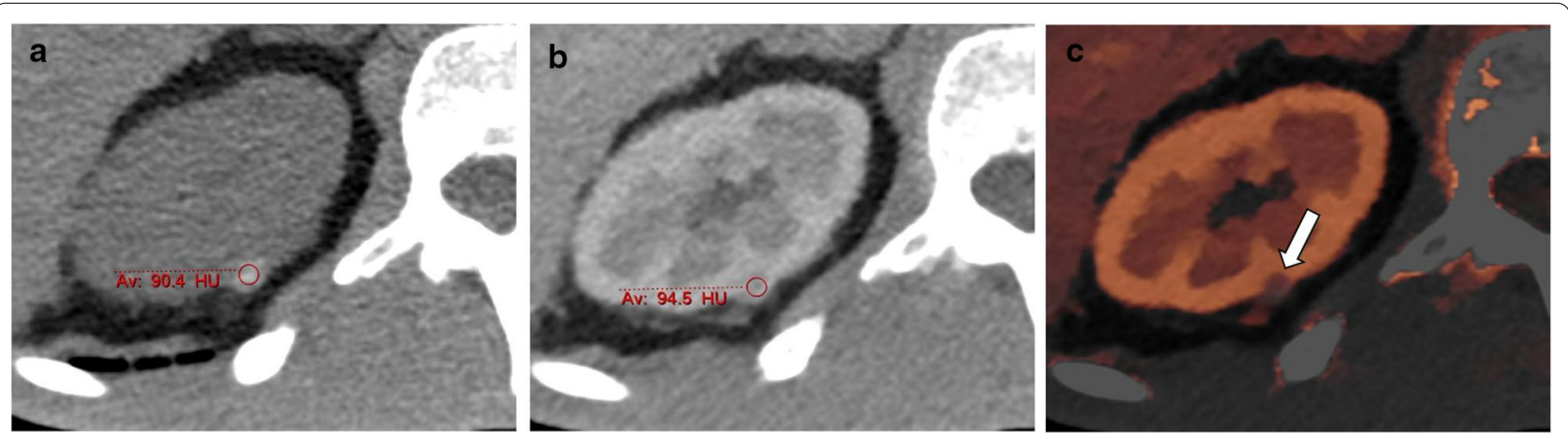

Fig. 4 An incidentally identified hyperdense right renal cyst in a 72-year-old man. Axial TNC image shows a markedly hyperdense cyst, most consistent with a hemorrhagic cyst (a) without enhancement on the portovenous phase image (b). However, iodine overlay maps show suspected mild enhancement (c). The iodine content of the cyst was $1.3 \mathrm{mg} / \mathrm{mL}$, which by accepted thresholds would be considered an enhancing lesion, however, the normalized iodine content was only $0.27 \mathrm{mg} / \mathrm{mL}$, consistent with lack of enhancement seen on conventional images 

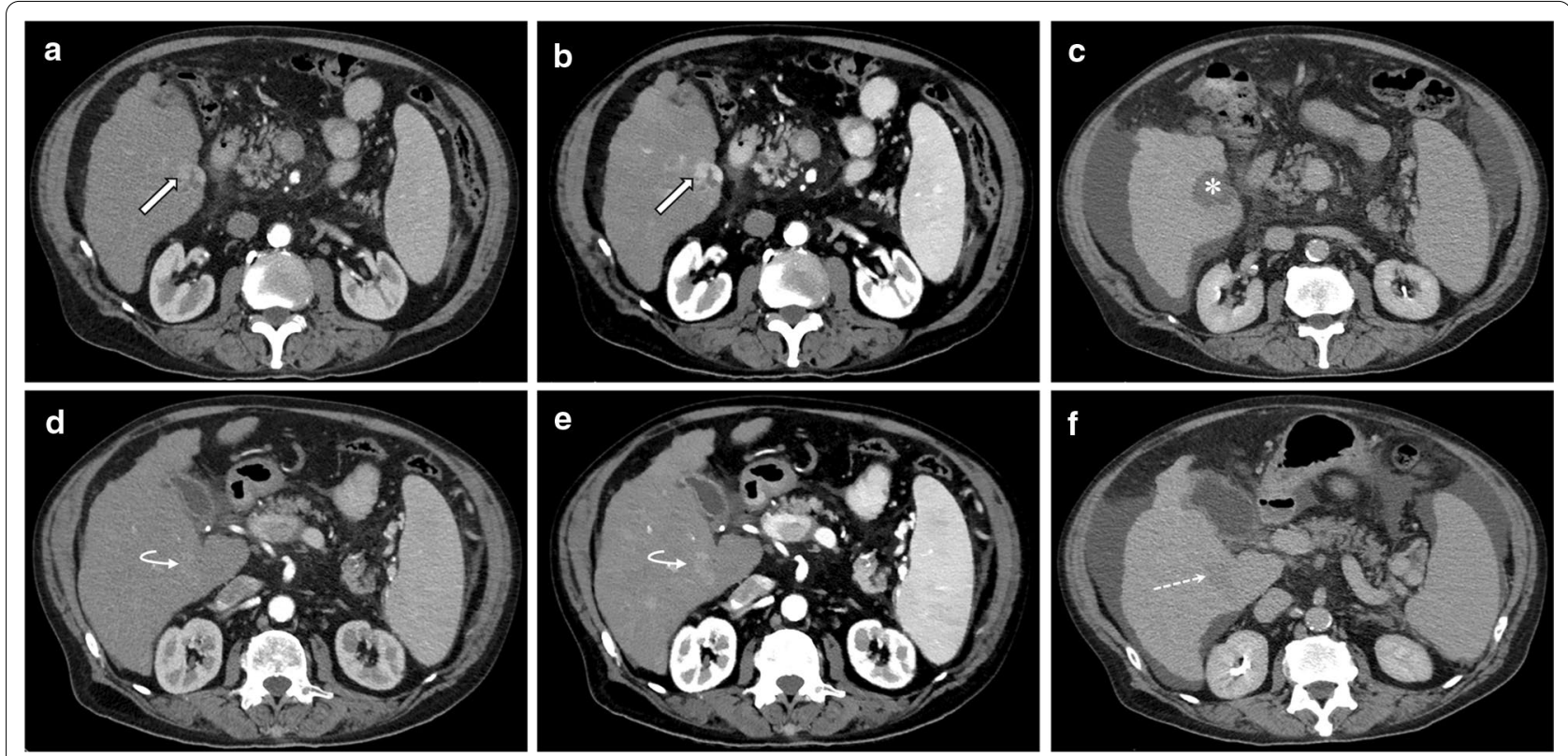

Fig. 5 Multiphasic spectral CT of a 73-year-old man with known HCC. The known tumor (arrows) shows typical hypervascularity on the conventional arterial phase (a) which is more prominent on $50 \mathrm{keV}$ mono-energetic image (b), and was ablated with good treatment response (asterisk) on the 1-month follow-up (c). However, there was an additional lesion (curved arrow) which should have been suspected by the radiologist on baseline imaging but was missed owing to its minimal arterial enhancement (d). This lesion would not have been missed if $50 \mathrm{keV}$ mono-energetic images had been reviewed prospectively (e), and on follow-up imaging showed definite washout and enlargement (dashed arrow), when the patient returned with advancing disease including tumor thrombus in the portal vein

using a threshold of 1.99 for iodine concentration normalized to the adjacent liver [45], although this still needs further verification before implementation in clinical practice. Iodine concentration in the equilibrium phase has been proposed as a method for the noninvasive evaluation of fibrosis based on the expansion of the interstitial space seen in cirrhosis [46].

Hepatic steatosis can also be diagnosed using material decomposition, and fat can be quantified accurately using this method [47-52].

\section{Pancreas}

Pancreatic adenocarcinoma is often difficult to visualize on conventional CT images, with only a vague hypodensity visible even on pancreatic phase images. Low keV spectral images and iodine quantification have been shown to improve the detection of pancreatic adenocarcinoma (Fig. 6) and also improve evaluation of arterial involvement [53-56].

Pancreatitis has also been studied, and in acute pancreatitis iodine quantification may aid in diagnosis, using a threshold of $2.1 \mathrm{mg} / \mathrm{mL}$ on pancreatic phase images [57] and also in assessing vascular complications and necrosis, using low keV images [58]. Differentiating chronic mass forming pancreatitis from adenocarcinoma is more problematic, but iodine quantification also shows promise in this regard [59]. In patients with chronic pancreatitis VNC images may aid in detecting pancreatic calcifications and ductal calculi on single phase contrast enhanced scans, although theoretically these may underestimate small calcifications because of erroneous calcium subtraction $[58,60]$.

\section{Biliary system}

Gallstones are often not identified on conventional CT, with more than half being radiolucent [60]. Thus, patients who undergo CT scanning for various reasons often require further workup on ultrasound or magnetic resonance cholangiopancreatography (MRCP), or even diagnostic endoscopic retrograde cholangiopancreatography (ERCP), depending on the clinical scenario. If $\mathrm{CT}$ could visualize cholelithiasis and choledocholithiasis reliably, there will be significant savings in cost and time-todiagnosis. Spectral material decomposition images detect non-calcified stones with high accuracy, because stones have a slightly lower effective atomic number (Z-effective) than bile (Fig. 7), and have a lower attenuation than bile on low $\mathrm{keV}$ images, optimally seen on $40 \mathrm{keV}$, and higher attenuation than bile on high $\mathrm{keV}$ images, optimally $140 \mathrm{keV}[60,61]$.

Acute cholecystitis is usually first evaluated on ultrasound, although large variability in sensitivity is seen 

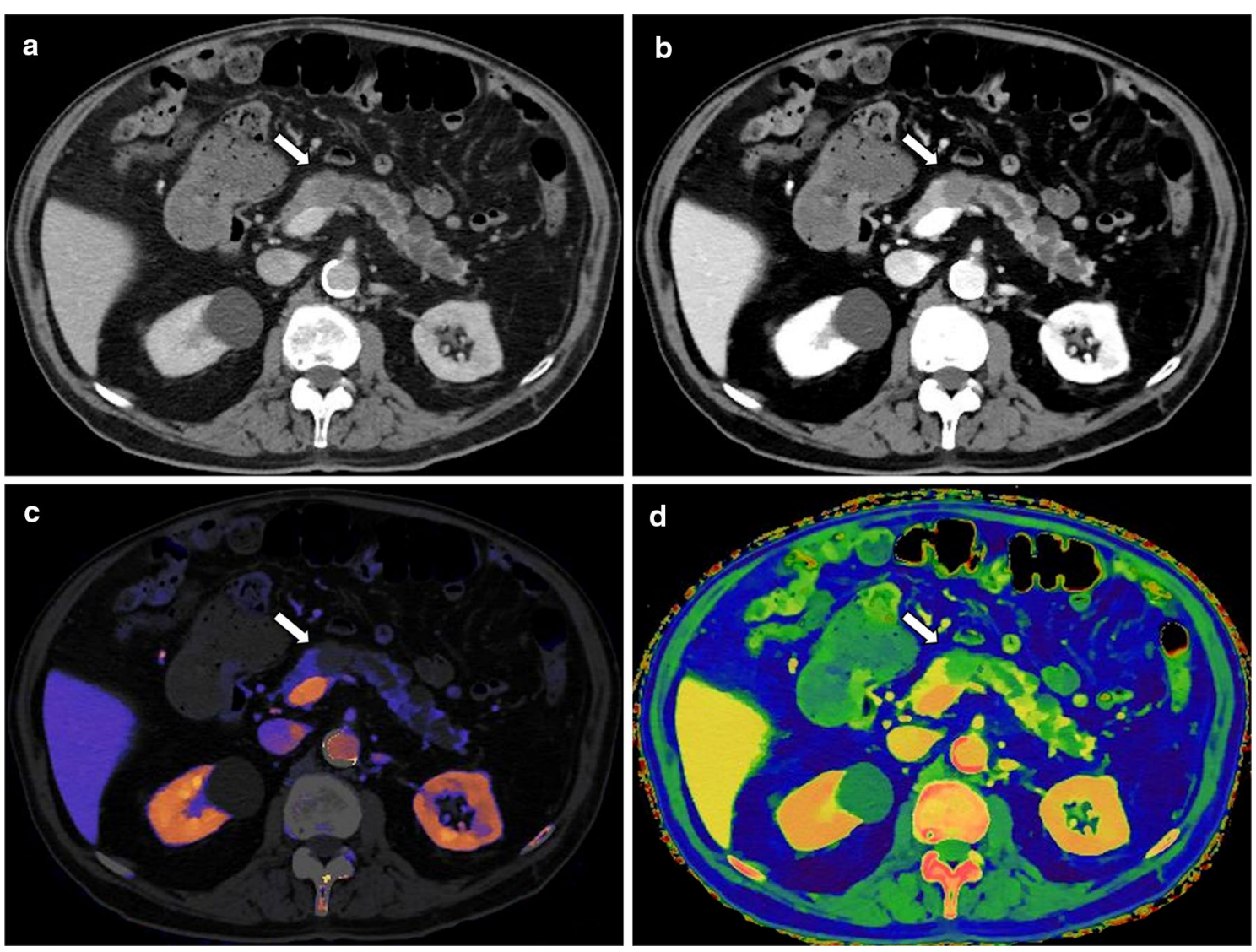

Fig. 6 Pancreatic adenocarcinoma in an 84-year-old man. On the late arterial phase image there is a lesion in the pancreatic body showing mild hypoenhancement compared to the normal pancreatic tissue (a). The lesion's conspicuity is markedly improved using 50 keV mono-energetic images (b), iodine concentration overlay maps (c), and effective atomic number maps (d)

with ultrasound [62], because of its wide availability and better visualization of gallstones, in conjunction with the ability to evaluate for a sonographic Murphy sign. However, studies have shown that CT in fact has better sensitivity [62, 63]. Spectral CT using low keV images and iodine maps may even improve the sensitivity for diagnosis, and can better evaluate suspected gangrenous cholecystitis because of better visualization of gallbladder wall enhancement abnormalities [60].

\section{Kidneys}

Incidental renal lesions are common, seen in approximately $10 \%$ of CT scans. While most are simple cysts, it is often difficult to distinguish between benign complicated cysts, possibly malignant complicated cysts and solid lesions on a single phase contrast enhanced study. This diagnostic dilemma leads to many follow up studies both on CT and MR. Spectral CT may enable accurate evaluation of some of these lesions, thus decreasing diagnostic costs and time. This has been one of the earliest applications of spectral CT, due to the high incidence of indeterminate renal incidentalomas [26, 34, 64-68]. Spectral CT can differentiate non-enhancing from enhancing lesions on single-phase scans (Fig. 8) using VNC reconstructions and also iodine maps and spectral attenuation curves [69], with the diagnosis of avidly enhancing lesions such as clearcell renal cell carcinoma (RCC) being easier than the less avidly enhancing papillary RCC. The threshold for determining enhancement of a lesion has been studied, and differences have been found between different spectral techniques and different scan timings (arterial vs. nephrographic) [34, 64]. For rapid $\mathrm{kV}$ switching, the reported thresholds are $1.22-2.0 \mathrm{ng} / \mathrm{mL}$, while for dual source scanners the reported thresholds are $0.5-1.3 \mathrm{ng} /$ $\mathrm{mL}$. Dual layer scanners thresholds have not been published yet. Normalizing the thresholds to the aorta's measured iodine concentration reduces intra-manufacturer variability, with a threshold of 0.3 across platforms [34]. Effective atomic number can also be used to diagnose enhancing masses, with an optimal threshold of 8.36 yielding a diagnostic accuracy of $86.6 \%[66]$. 

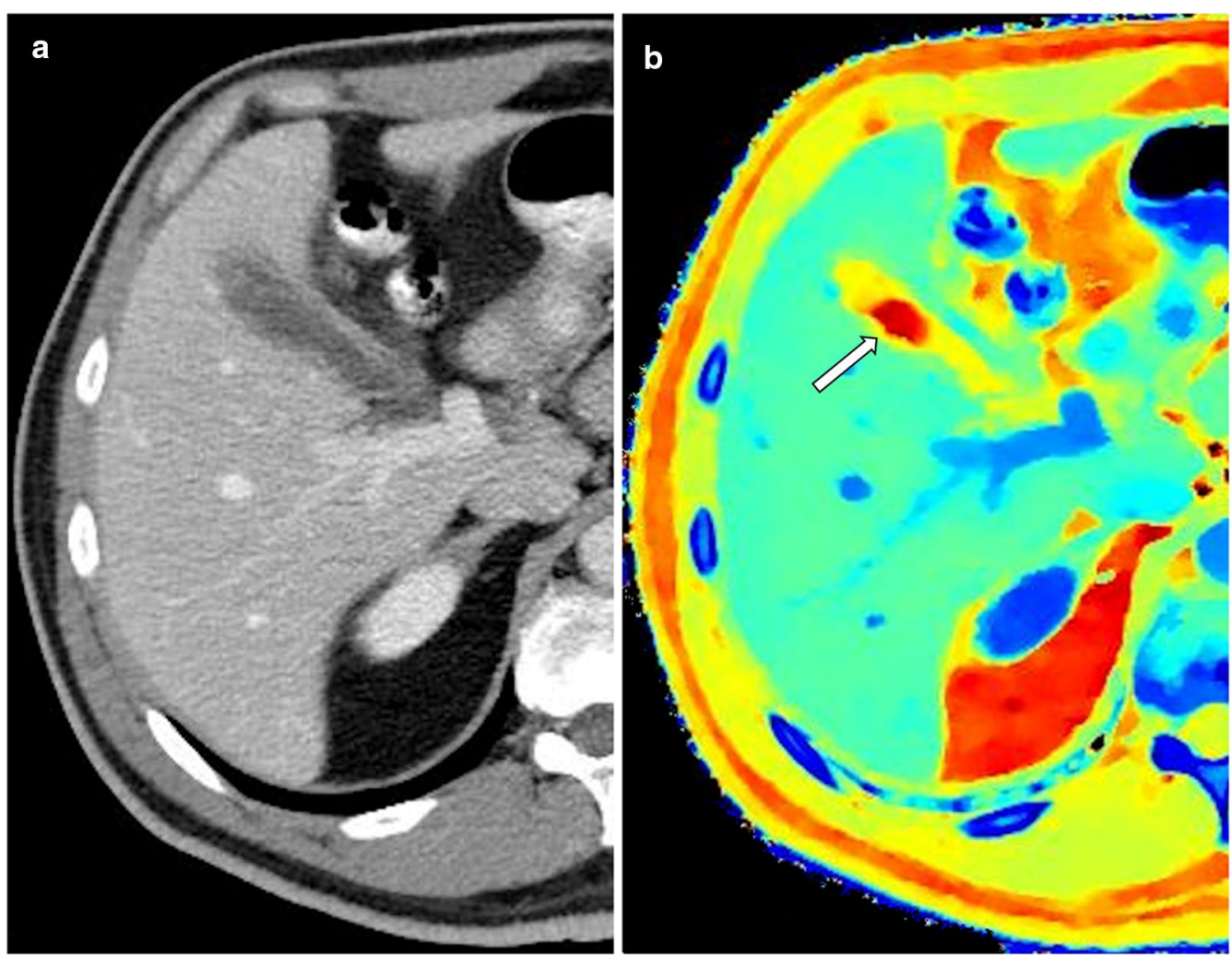

Fig. 7 CT of a 64-year-old man showing mild heterogeneity of the gallbladder contents on conventional images (a), with a clear gallbladder stone visible on the effective atomic number map (b) as a focal area with lower atomic number than surrounding bile (arrow)
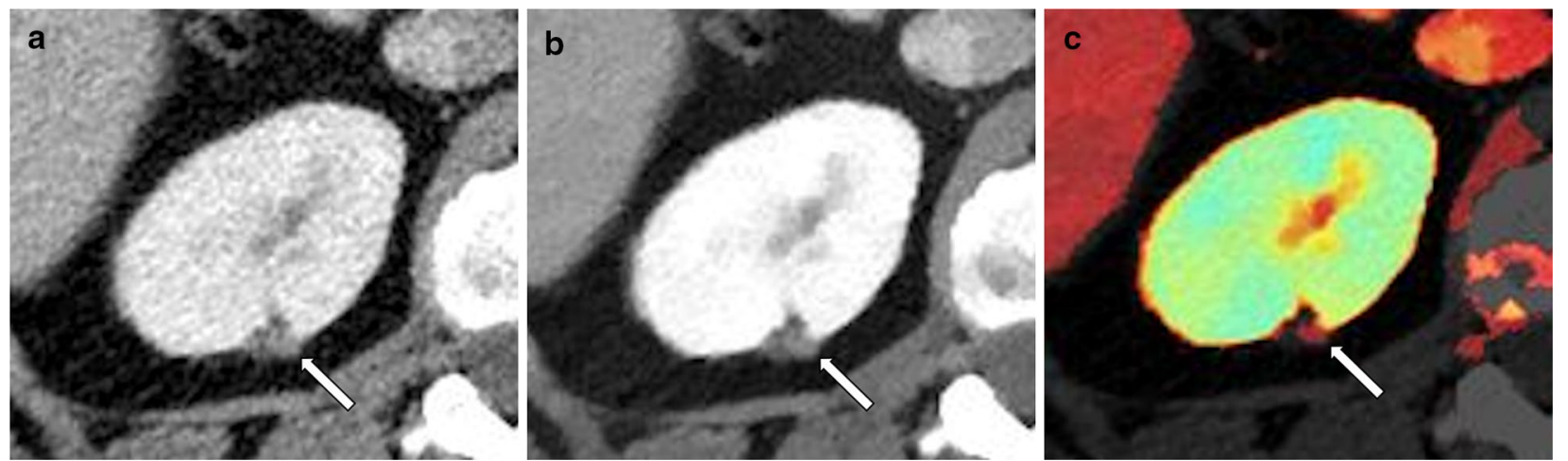

Fig. 8 A 54-year-old woman underwent a routine abdominal CT before hernioplasty. Conventional images showed an incidental finding in the right kidney (arrows) with heterogeneous attenuation on the portovenous phase (a). The lesion's heterogeneity and suspected enhancing component are accentuated using a $50 \mathrm{keV}$ mono-energetic image $(\mathbf{b})$. lodine maps prove that the lesion's high attenuation is true enhancement (c), showing iodine content of $4.8 \mathrm{mg} / \mathrm{mL}$ and a normalized iodine content of $1.1 \mathrm{mg} / \mathrm{mL}$, suspicious for RCC

Measuring iodine concentration is at least as accurate as measuring enhancement values on enhanced images compared with unenhanced images $[65,67,68]$, but the effect of pseudo-enhancement on accurate measurements must still be considered as it is with conventional CT [70, 71]. This is most pronounced in small lesions, with a diameter under $8 \mathrm{~mm}$, which is why there is likely still a size threshold for reliably evaluating renal lesions, even with spectral imaging. In these cases VNC has similar limitations when there is insufficient iodine removal close to the collecting system or when there is avid enhancement of the renal parenchyma [69]. 
Iodine concentration is also showing promise in differentiating between histological sub-types of RCC, and has been shown to accurately differentiate between clearcell RCC and papillary RCC, using a threshold of $0.9 \mathrm{mg} /$ $\mathrm{mL}$ with an overall accuracy of $95.3 \%$, while also showing promise in determining tumor grade [72].

Low $\mathrm{keV}$ mono-energetic images can also be used to decrease the amount of contrast used in CT urography, with adequate enhancement achieved using $50 \%$ of contrast material dose both for urographic phase evaluation and for blood vessel evaluation prior to surgery [73].

Evaluation of nephrolithiasis has also been markedly improved with spectral imaging. Uric acid stones, which are often treated medically, can be reliably distinguished from other types of stones (Fig. 9), which may otherwise necessitate an invasive approach, with an accuracy ranging between 90 to $100 \%$ [58, 60, 74, 75]. More advanced material decomposition using dual source systems with tin filtration, or advanced applications of spectral detectors, can also differentiate between most other types of stones $[60,76]$, although this has a lesser clinical impact.

\section{Adrenals}

Adrenal incidentalomas, seen in $1-4.2 \%$ of the scanned population, are considered a major cause of further imaging required for incidental lesions, and have widely acceptable imaging guidelines on conventional CT [77]. Adenomas can be diagnosed on conventional CT using TNC images, with a threshold of $10 \mathrm{HU}$ for differentiating between adenomas and other lesions, and with dedicated adrenal scans using washout calculations [77]. However, single phase enhanced routine abdominal scans cannot make this differentiation, leading to further imaging. Spectral imaging has the potential to obviate further imaging, both by enabling diagnosis on single phase enhanced CT scans, and by improving the diagnosis of lesions considered to be indeterminate, using current $\mathrm{CT}$ and MRI criteria.

Spectral VNC images can be used instead of TNC, although the minimal difference in $\mathrm{HU}$ measurements between VNC and TNC [11, 78, 79] leads to altered sensitivity and specificity when using the same threshold, and the optimal VNC threshold for diagnosing an adenoma has not been determined yet. However, it can be noted that no false positive cases were found when using the same threshold [80]. This implies this threshold can be used to safely diagnose adenomas, with the risk of calling more lesions indeterminate compared to TNC (Fig. 2). Studies have also shown differences in attenuation of adrenal lesions on VNC compared to TNC images, reported more when scans were performed early in the portal-venous phase compared to later in the portal-venous phase [80]. Therefore, reaching a widely accepted threshold for diagnosing adenomas on VNC using routine abdominal scans which are likely to be performed with slightly different scan timings in different institutions may prove to be difficult.

Studies have also demonstrated the superiority of spectral derived data other than $\mathrm{VNC}$, using attenuation curves at different $\mathrm{keV}$, material decomposition using fatwater and fat-iodine pairs, z-effective and other parameters $[26,64,81]$. These studies need further corroboration using larger cohorts and multiple spectral technologies, before the results can be used in clinical practice.

\section{Gastrointestinal tract}

The obvious application in evaluating bowel disease is the evaluation of active hemorrhage. Low $\mathrm{keV}$ images accentuate active extravasation and may facilitate detection of intraluminal hemorrhage (Fig. 10) [60, 82]. The use of VNC images in lieu of TNC images also has the potential to reduce radiation in active gastrointestinal hemorrhage protocols [40] and allow for confident detection of hemorrhage on non-dedicated portal-venous phase only scans.

Acute ischemia may also be better evaluated on spectral CT, in both acute mesenteric occlusion [83, 84] and in ischemia secondary to bowel obstruction $[85,86]$. Although one study has only been able to show increased diagnostic confidence, without improved diagnostic performance [86], the other studies have shown improved performance for detection of abnormally enhancing bowel segments using iodine maps and low $\mathrm{keV}$ images.

Active bowel inflammation severity in Crohn's disease has been shown to be related to iodine concentration [87, 88] and spectral HU curves [88]. Qualitative assessment of active inflammation may also be improved because of the heightened hyper-enhancement, seen on low $\mathrm{keV}$ images (Fig. 11). The most useful low keV setting for diagnosis of active inflammation was found to be $40 \mathrm{keV}$ (the lowest achievable in the study) [89], surprising considering the fact that this $\mathrm{keV}$ also has the greatest amount of noise and is not routinely used in clinical practice in most centers [86]. Acute appendicitis, which is another type of inflammation, can also become more visible using iodine maps and low keV images [60] and these images also improve diagnostic performance in cases of gangrenous appendicitis [90].

CT colonography uses low-dose CT to evaluate the colon for polyps, and electronic fecal cleansing is often used to correct for inadequate bowel preparation, by using fecal tagging with iodine or barium. Electronic cleansing often causes artifacts which decrease image quality. Spectral data allows for better electronic cleansing and fecal tagging, with improved image quality $[91,92]$. Deep learning is now being used to 

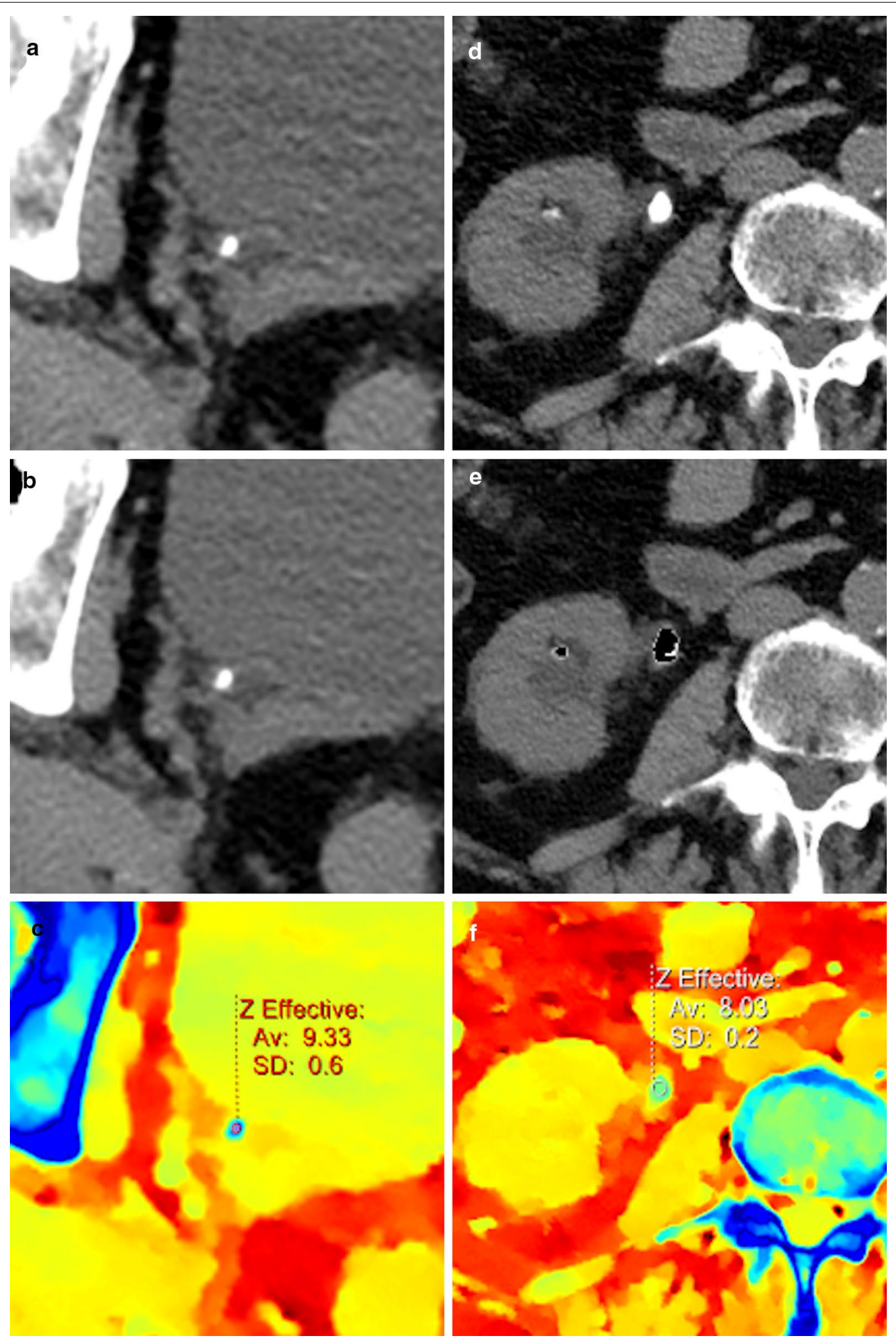

Fig. 9 Side-by-side images showing uric acid evaluation in two different patients evaluated for nephrolithiasis. a-c showing a calcium-containing stone in the distal ureter, $(\mathbf{d}-\mathbf{f})$ showing uric acid stones in the lower pole calix and proximal ureter, proven as uric acid stones by laboratory evaluation following lithotripsy and stenting. $\mathbf{a}$, d conventional anatomic images, (b, e) "uric acid removed" overlay images, on which uric acid is removed from the image leaving a black pixels, and $(\mathbf{c}, \mathbf{f})$ are atomic number images showing the different atomic numbers of the different types of stones 

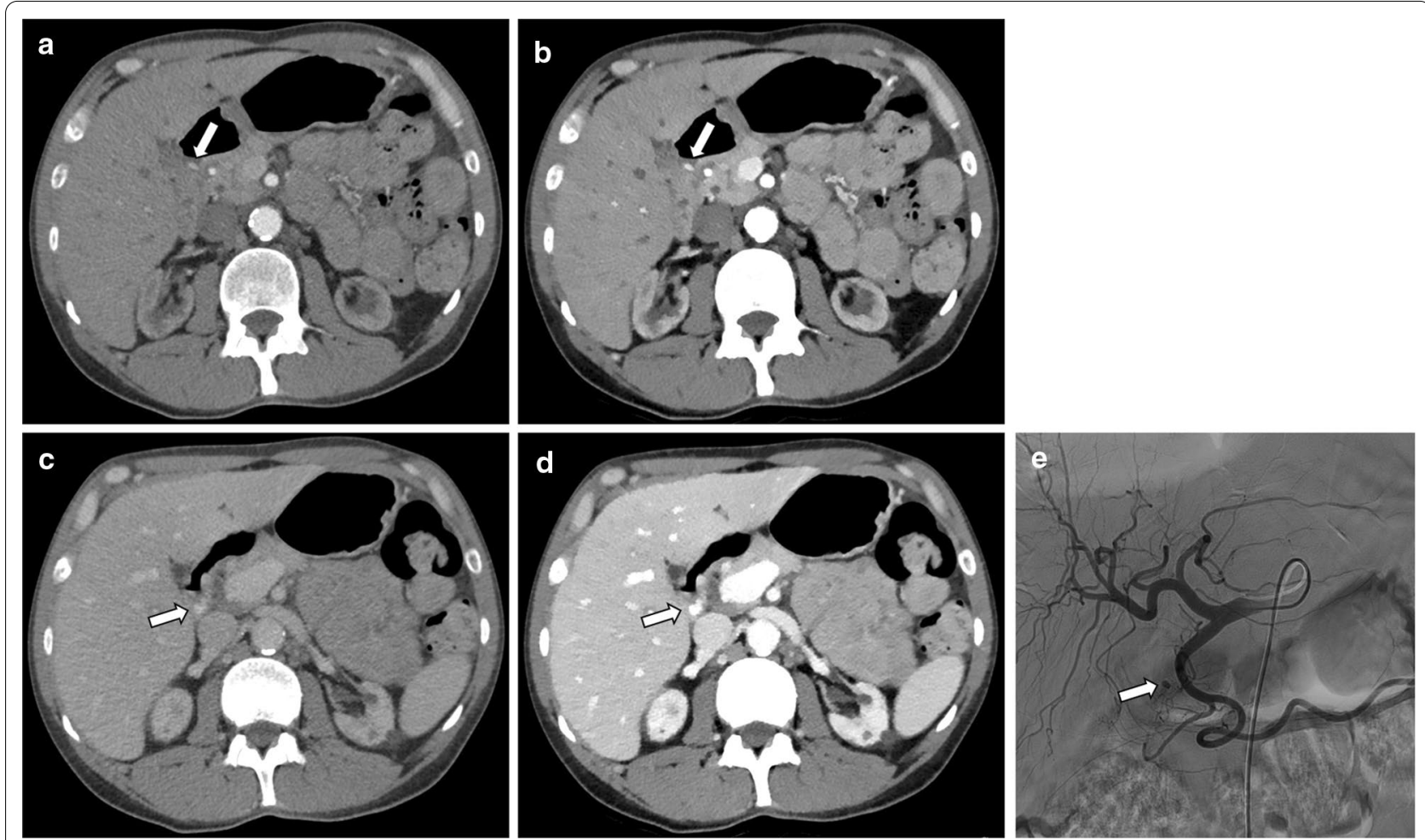

Fig. 10 A 40-year-old man with upper gastrointestinal bleeding who underwent multiphasic CT for localization. Conventional images show contrast extravasation in the duodenal bulb (arrows) on the arterial (a) and venous (c) phases. The extravasation is more conspicuous using 50 keV mono-energetic images on both the arterial $(\mathbf{b})$ and venous (d) phases. The focus of active bleeding is proven on digital subtraction angiography (e)
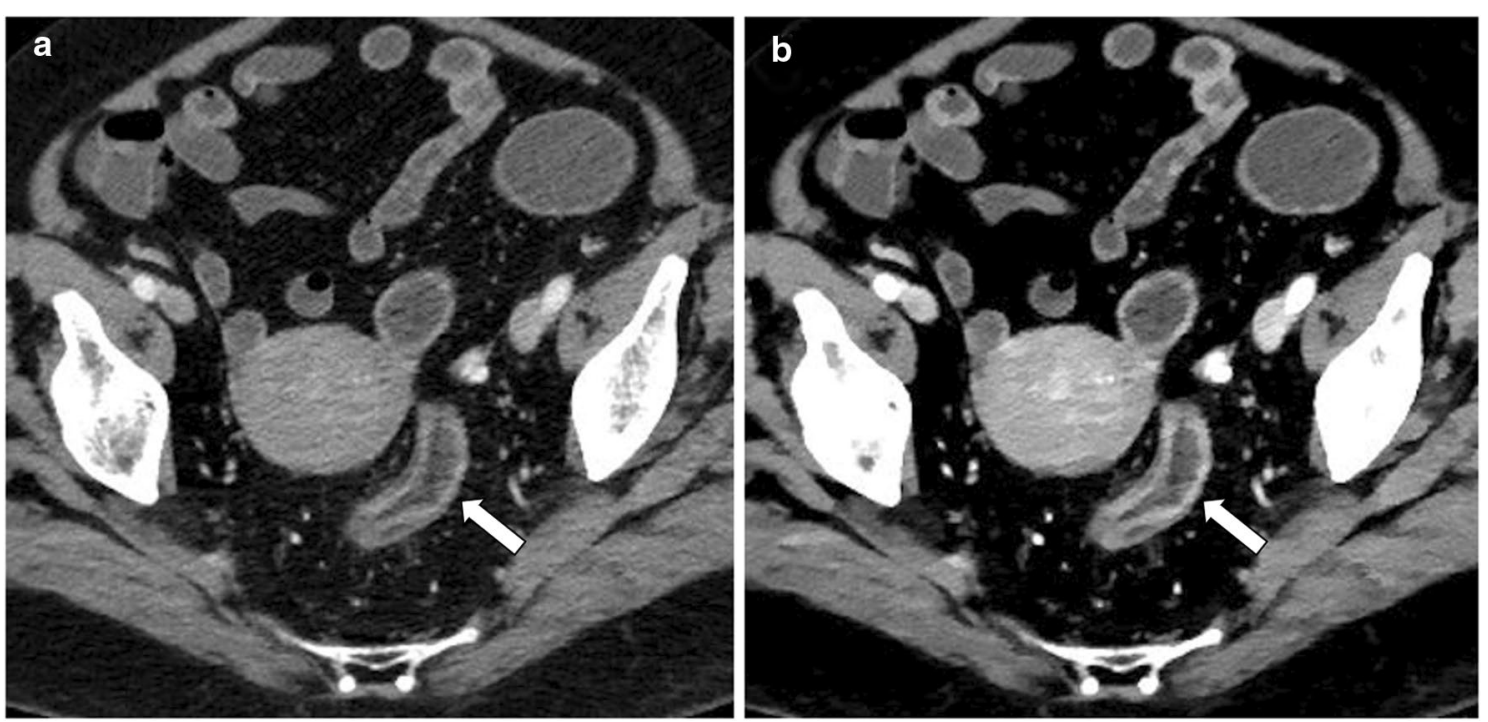

Fig. 11 CT enterography of a 74-year-old woman with known Crohn's disease of the colon. Conventional images show mild mucosal hyperenhancement in the sigmoid colon (a) which is more conspicuous using $50 \mathrm{keV}$ mono-energetic images (b) 
improve cleansing algorithms, and has been shown to work better when using spectral data [93].

Bowel tumor evaluation on spectral CT is also being investigated. The obvious advantages lie in the detection of primary or metastatic hyper-enhancing tumors, such as neuroendocrine tumors and melanoma metastases using low $\mathrm{KeV}$ mono-energetic images and iodine maps [94]. Spectral data is also showing promise in determining histological origin and grade, for instance determining the histological origin of ampullary tumors [95], differentiating between bowel adenocarcinoma and lymphoma [96], differentiating squamous cell vs. adenocarcinoma of the gastroesophageal junction [97], differentiating high vs low-risk gastrointestinal stromal tumors (GIST) [98], diagnosing colorectal carcinomas with microsatellite instability [50] or high grade characteristics [99]. Differentiating metastatic lymph nodes from benign lymph nodes may also be possible using z-effective and iodine concentrations, with accuracy similar to MRI on one study, and this has been studied mostly for rectal carcinoma but also gastric cancers [100-102]. Response to treatment may also be better evaluated with spectral data, as shown in rectal tumors [103] and in GIST [64].

\section{Genital system}

Although not extensively studied, the same basic principles as in other organs apply to the genital system. A major possible application is differentiation between benign and malignant tumors. Iodine maps and low $\mathrm{KeV}$ images improve the identification of enhancing septations, which raise the suspicion for malignancy [104]. A recent study found an iodine content threshold of $0.9 \mathrm{mg} / \mathrm{mL}$ to have an $81 \%$ sensitivity and a $73 \%$ specificity in distinguishing benign from malignant tumors. It also found that a Z-effective threshold of 8.16 had an $85 \%$ sensitivity and a $73 \%$ specificity [105]. For assessment of depth of myometrial invasion of endometrial carcinoma, low $\mathrm{KeV}$ images perform comparably to trans-vaginal ultrasound, when compared with pathology, with a $91 \%$ sensitivity, $100 \%$ specificity and $94 \%$ accuracy, with conventionalequivalent images showing only a $57 \%$ sensitivity, $86 \%$ specificity and $71 \%$ accuracy [106]. In cervical malignancies, iodine maps are showing promise in evaluating response to chemo-radiation therapy and in predicting outcome [107]. Iodine content has also shown promise in differentiating cervical carcinoma involvement of lymph nodes from normal or reactive lymph nodes [108], with metastatic lymph nodes showing significantly lower iodine content, although no definitive thresholds exist.

\section{Conclusions and future developments}

Currently, the most well established application of spectral imaging is the use of mono-energetic images of high and low $\mathrm{KeV}$ for better visualization of enhancing structures, decreasing contrast media volume and decreasing metal artifacts. VNC and z-effective evaluation of specific materials are also relatively robust and can be used clinically in certain settings, but need further improvement before widely adopted, in our opinion. Using spectral data for lesion characterization is very promising, however, we believe there is not enough knowledge to create a clinically robust threshold for iodine content. There is also variability derived from the different hardware and algorithms used by the different vendors, possibly precluding our ability to reach cross-platform thresholds. This application will likely become well established for clinical use only when such universal thresholds are determined.

The future of spectral CT is right beyond the bend, with photon-counting systems already being developed and site-tested. These scanners are expected to be available clinically in the next few years, and are expected to improve material specificity, energy separation and likely also inter-vendor variability.

\section{Abbreviations \\ CNR: Contrast-to-noise ratio; CT: Computed tomography; ERCP: Endoscopic retrograde cholangiopancreatography; GIST: Gastrointestinal stromal tumors; HCC: Hepatocellular carcinoma; HU: Hounsfield units; keV: Kilo-electron Volt; kV: Kilo-Volt; kVp: Peak tube voltage; MRCP: Magnetic resonance cholangio- pancreatography; MRI: Magnetic Resonance Imaging; NET: Neuroendocrine tumor; RCC: Renal cell carcinoma; SNR: Signal-to-noise ratio; TNC: True non- contrast; VNC: Virtual non-contrast.}

\section{Authors' contributions}

SZA performed literature search, was the main contributor in writing the manuscript and prepared the figures and legends. AR prepared the figures and legends and contributed to the manuscript. RK performed literature search and contributed to the manuscript. $A B$ assisted in the design of the review and contributed to the manuscript.

\section{Funding}

Not applicable (this is a review, not original research, and therefore no funding was necessary).

\section{Availability of data and materials}

Not applicable (this is a review, not original research).

\section{Declarations}

Ethics approval and consent to participate

Not applicable.

\section{Consent for publication}

Not applicable.

\section{Competing interests}

The authors declare that they have no competing interests. 


\author{
Author details \\ University, Tel Aviv, Israel. \\ Received: 30 January 2021 Accepted: 16 August 2021 \\ Published online: 27 September 2021
}

${ }^{1}$ Department of Diagnostic Radiology, Tel Aviv Sourasky Medical Center, 6 Weizmann St., 6423906 Tel Aviv, Israel. ${ }^{2}$ Sackler School of Medicine, Tel Aviv

\section{References}

1. Krauss B (2018) Dual-energy computed tomography: technology and challenges. Radiol Clin North Am 56:497-506. https://doi.org/10.1016/j. rcl.2018.03.008

2. Jacobsen M, Schellingerhout D, Wood C et al (2017) Intermanufacturer comparison of dual-energy CT iodine quantification and monochromatic attenuation: a phantom study. Radiology 287:170896. https://doi. org/10.1148/radiol.2017170896

3. Megibow AJ, Kambadakone A, Ananthakrishnan L (2018) Dual-energy computed tomography: image acquisition, processing, and workflow. Radiol Clin North Am 56:507-520. https://doi.org/10.1016/j.rcl.2018.03. 001

4. van Ommen F, de Jong HWAM, Dankbaar JW et al (2019) Dose of CT protocols acquired in clinical routine using a dual-layer detector CT scanner: a preliminary report. Eur J Radiol 112:65-71. https://doi.org/10. 1016/j.ejrad.2019.01.011

5. Siegel MJ, Mhlanga JC, Salter A, Ramirez-Giraldo JC (2021) Comparison of radiation dose and image quality between contrast-enhanced single- and dual-energy abdominopelvic computed tomography in children as a function of patient size. Pediatr Radiol. https://doi.org/10. 1007/s00247-021-05127-3

6. Forghani R, De Man B, Gupta R (2017) Dual-energy computed tomography: physical principles, approaches to scanning, usage, and implementation: part 1. Neuroimaging Clin N Am 27:371-384. https:// doi.org/10.1016/j.nic.2017.03.002

7. Jamali S, Michoux N, Coche E, Dragean CA (2019) Virtual unenhanced phase with spectral dual-energy CT: is it an alternative to conventional true unenhanced phase for abdominal tissues? Diagn Interv Imaging 100:503-511. https://doi.org/10.1016/j.diii.2019.04.007

8. Parakh A, Macri F, Sahani D (2018) Dual-energy computed tomography: dose reduction, series reduction, and contrast load reduction in dual-energy computed tomography. Radiol Clin North Am 56:601-624. https://doi.org/10.1016/j.rcl.2018.03.002

9. Ananthakrishnan L, Rajiah P, Ahn R et al (2017) Spectral detector CTderived virtual non-contrast images: comparison of attenuation values with unenhanced CT. Abdom Radiol (NY) 42:702-709. https://doi.org/ 10.1007/s00261-016-1036-9

10. Durieux P, Gevenois PA, Van MA et al (2018) Abdominal attenuation values on virtual and true unenhanced images obtained with third-generation dual-source dual-energy CT. AJR Am J Roentgenol 210:1042-1058. https://doi.org/10.2214/AJR.17.18248

11. Slebocki K, Kraus B, Chang D-H et al (2017) Incidental Findings in Abdominal Dual-Energy Computed Tomography: Correlation Between True Noncontrast and Virtual Noncontrast Images Considering Renal and Liver Cysts and Adrenal Masses. J Comput Assist Tomogr 41:294-297. https://doi.org/10.1097/RCT.0000000000000503

12. Grosse Hokamp N, Gilkeson R, Jordan MK et al (2019) Virtual monoenergetic images from spectral detector CT as a surrogate for conventional CT images: Unaltered attenuation characteristics with reduced image noise. Eur J Radiol 117:49-55. https://doi.org/10.1016/j.ejrad.2019.05. 019

13. Atwi NE, Smith DL, Flores CD et al (2019) Dual-energy CT in the obese: a preliminary retrospective review to evaluate quality and feasibility of the single-source dual-detector implementation. Abdom Radiol (NY) 44:783-789. https://doi.org/10.1007/s00261-018-1774-y

14. Matsumoto K, Jinzaki M, Tanami Y et al (2011) Virtual monochromatic spectral imaging with fast kilovoltage switching: improved image quality as compared with that obtained with conventional 120-kVp CT. Radiology 259:257-262. https://doi.org/10.1148/radiol.11100978

15. Wei L, Li S, Gao Q et al (2016) Use of low tube voltage and low contrast agent concentration yields good image quality for aortic CT angiography. Clin Radiol 71:1313.e5-1313.e10. https://doi.org/10.1016/j. crad.2016.07.018

16. Higashigaito K, Schmid T, Puippe G et al (2016) CT Angiography of the Aorta: Prospective Evaluation of Individualized Low-Volume Contrast Media Protocols. Radiology 280:960-968. https://doi.org/10.1148/radiol. 2016151982

17. Ippolito D, Talei Franzesi C, Fior D et al (2015) Low kV settings CT angiography (CTA) with low dose contrast medium volume protocol in the assessment of thoracic and abdominal aorta disease: a feasibility study. Br J Radiol 88:20140140. https://doi.org/10.1259/bjr.20140140

18. Chung YE, You JS, Lee $\mathrm{H}$-J et al (2015) Possible contrast media reduction with low keV monoenergetic images in the detection of focal liver lesions: a dual-energy CT animal study. PLoS One 10:e0133170. https:// doi.org/10.1371/journal.pone.0133170

19. Shuman WP, O'Malley RB, Busey JM et al (2017) Prospective comparison of dual-energy CT aortography using $70 \%$ reduced iodine dose versus single-energy CT aortography using standard iodine dose in the same patient. Abdom Radiol (NY) 42:759-765. https://doi.org/10.1007/ s00261-016-1041-z

20. Hickethier T, Kroeger JR, Lennartz S et al (2019) Venous-phase chest CT with reduced contrast medium dose: Utilization of spectral low keV monoenergetic images improves image quality. Eur J Radiol 122:108756. https://doi.org/10.1016/j.ejrad.2019.108756

21. Noda Y, Goshima S, Nakashima Y et al (2019) lodine dose optimization in portal venous phase virtual monochromatic images of the abdomen: Prospective study on rapid kVp switching dual energy CT. Eur J Radiol 122:108746. https://doi.org/10.1016/j.ejrad.2019.108746

22. Tsang DS, Merchant TE, Merchant SE et al (2017) Quantifying potential reduction in contrast dose with monoenergetic images synthesized from dual-layer detector spectral CT. Br J Radiol 90:20170290. https:// doi.org/10.1259/bjr.20170290

23. Clark ZE, Bolus DN, Little MD, Morgan DE (2015) Abdominal rapid-kVpswitching dual-energy MDCT with reduced IV contrast compared to conventional MDCT with standard weight-based IV contrast: an intrapatient comparison. Abdom Imaging 40:852-858. https://doi.org/10. 1007/s00261-014-0253-3

24. Parakh A, Negreros-Osuna AA, Patino M et al (2019) Low-keV and Low-kVp CT for Positive Oral Contrast Media in Patients with Cancer: A Randomized Clinical Trial. Radiology 291:620-629. https://doi.org/10. 1148/radiol.2019182393

25. Patino M, Murcia DJ, lamurri AP et al (2017) Impact of low-energy CT imaging on selection of positive oral contrast media concentration. Abdom Radiol (NY) 42:1298-1309. https://doi.org/10.1007/ s00261-016-0993-3

26. Fulton N, Rajiah P (2018) Abdominal applications of a novel detectorbased spectral CT. Curr Probl Diagn Radiol 47:110-118. https://doi.org/ 10.1067/j.cpradiol.2017.05.001

27. Wellenberg RHH, Boomsma MF, van Osch JAC et al (2017) Quantifying metal artefact reduction using virtual monochromatic dual-layer detector spectral CT imaging in unilateral and bilateral total hip prostheses. Eur J Radiol 88:61-70. https://doi.org/10.1016/j.ejrad.2017.01.002

28. Hakvoort ET, Wellenberg RHH, Streekstra GJ (2019) Quantifying near metal visibility using dual energy computed tomography and iterative metal artifact reduction in a fracture phantom. Phys Med 69:9-18. https://doi.org/10.1016/j.ejmp.2019.11.006

29. Lu X, Lu Z, Yin J, et al (2019) Effects of radiation dose levels and spectral iterative reconstruction levels on the accuracy of iodine quantification and virtual monochromatic CT numbers in dual-layer spectral detector CT: an iodine phantom study. Quant Imaging Med Surg 9:188-200. https://doi.org/10.21037/qims.2018.11.12

30. Kim H, Park CM, Kang CK et al (2018) Effect of CT acquisition parameters on iodine density measurement at dual-layer spectral CT. AJR Am J Roentgenol 211:748-754. https://doi.org/10.2214/AJR.17.19381

31. Wortman JR, Sodickson AD (2018) Pearls, pitfalls, and problems in dualenergy computed tomography imaging of the body. Radiol Clin North Am 56:625-640. https://doi.org/10.1016/j.rcl.2018.03.007

32. Jacobsen MC, Cressman ENK, Tamm EP et al (2019) Dual-energy CT: lower limits of iodine detection and quantification. Radiology 292:414-419. https://doi.org/10.1148/radiol.2019182870

33. Grosse Hokamp N, Abdullayev N, Persigehl T et al (2019) Precision and reliability of liver iodine quantification from spectral detector CT: 
evidence from phantom and patient data. Eur Radiol 29:2098-2106. https://doi.org/10.1007/s00330-018-5744-0

34. Patel BN, Vernuccio F, Meyer M et al (2019) Dual-energy CT material density iodine quantification for distinguishing vascular from nonvascular renal lesions: normalization reduces intermanufacturer threshold variability. AJR Am J Roentgenol 212:366-376. https://doi.org/10.2214/ AJR.18.20115

35. Boning G, Feldhaus F, Adelt $S$ et al (2019) Clinical routine use of virtual monochromatic datasets based on spectral CT in patients with hypervascularized abdominal tumors - evaluation of effectiveness and efficiency. Acta Radiol 60:425-432. https://doi.org/10.1177/0284185118 786077

36. Park JH, Kim SH, Park HS et al (2011) Added value of $80 \mathrm{kVp}$ images to averaged $120 \mathrm{kVp}$ images in the detection of hepatocellular carcinomas in liver transplantation candidates using dual-source dual-energy MDCT: results of JAFROC analysis. Eur J Radiol 80:e76-85. https://doi. org/10.1016/j.ejrad.2010.08.018

37. LV P, Lin XZ, Chen K, Gao J (2012) Spectral CT in patients with small HCC: investigation of image quality and diagnostic accuracy. Eur Radiol 22:2117-2124. https://doi.org/10.1007/s00330-012-2485-3

38. Anzidei M, Di Martino M, Sacconi B et al (2015) Evaluation of image quality, radiation dose and diagnostic performance of dual-energy CT datasets in patients with hepatocellular carcinoma. Clin Radiol 70:966-973. https://doi.org/10.1016/j.crad.2015.05.003

39. Grosse Hokamp N, Obmann VC, Kessner R et al (2018) Improved visualization of hypodense liver lesions in virtual monoenergetic images from spectral detector CT: Proof of concept in a 3D-printed phantom and evaluation in 74 patients. Eur J Radiol 109:114-123. https://doi.org/10. 1016/j.ejrad.2018.11.001

40. Sun H, Hou X-Y, Xue H-D et al (2015) Dual-source dual-energy CT angiography with virtual non-enhanced images and iodine map for active gastrointestinal bleeding: image quality, radiation dose and diagnostic performance. Eur J Radiol 84:884-891. https://doi.org/10.1016/j.ejrad. 2015.01.013

41. Ascenti G, Sofia C, Mazziotti S et al (2016) Dual-energy CT with iodine quantification in distinguishing between bland and neoplastic portal vein thrombosis in patients with hepatocellular carcinoma. Clin Radiol 71:938.e1-9. https://doi.org/10.1016/j.crad.2016.05.002

42. Su L, Hu L, Liang P et al (2019) Clinical efficacy of spectral computed tomography for evaluating liver function in patients with Budd-Chiari syndrome. Acad Radiol 26:461-466. https://doi.org/10.1016/j.acra.2018. 05.003

43. Wang N, Ju Y, Wu J et al (2019) Differentiation of liver abscess from liver metastasis using dual-energy spectral CT quantitative parameters. Eur J Radiol 113:204-208. https://doi.org/10.1016/j.ejrad.2019.02.024

44. Pfeiffer D, Parakh A, Patino M et al (2018) lodine material density images in dual-energy CT: quantification of contrast uptake and washout in HCC. Abdom Radiol (NY) 43:3317-3323. https://doi.org/10.1007/ s00261-018-1636-7

45. Gao L, Lv Y, Jin Y et al (2019) Differential diagnosis of hepatic cancerous nodules and cirrhosis nodules by spectral CT imaging: a feasibility study. Acta Radiol. https://doi.org/10.1177/0284185119840230

46. Bottari A, Silipigni S, Carerj ML et al (2019) Dual-source dual-energy CT in the evaluation of hepatic fractional extracellular space in cirrhosis. Radiol Med. https://doi.org/10.1007/s11547-019-01089-7

47. Hyodo T, Yada N, Hori M et al (2017) Multimaterial decomposition algorithm for the quantification of liver fat content by using fastkilovolt-peak switching dual-energy CT: clinical evaluation. Radiology 283:108-118. https://doi.org/10.1148/radiol.2017160130

48. Hyodo T, Hori M, Lamb P et al (2017) Multimaterial decomposition algorithm for the quantification of liver fat content by using Fast-Kilovolt-Peak switching dual-energy CT: experimental validation. Radiology 282:381-389. https://doi.org/10.1148/radiol.2016160129

49. Zheng X, Ren Y, Phillips WT et al (2013) Assessment of hepatic fatty infiltration using spectral computed tomography imaging: a pilot study. J Comput Assist Tomogr 37:134-141. https://doi.org/10.1097/RCT.0b013 e31827ddad3

50. Wu J, Lv Y, Wang $\mathrm{N}$ et al (2019) The value of single-source dual-energy CT imaging for discriminating microsatellite instability from microsatellite stability human colorectal cancer. Eur Radiol 29:3782-3790. https:// doi.org/10.1007/s00330-019-06144-5
51. Kramer H, Pickhardt PJ, Kliewer MA et al (2017) Accuracy of liver fat quantification with advanced $\mathrm{CT}, \mathrm{MRI}$, and ultrasound techniques: prospective comparison with MR spectroscopy. AJR Am J Roentgenol 208:92-100. https://doi.org/10.2214/AJR.16.16565

52. Zhang $\mathrm{YN}$, Fowler $\mathrm{KJ}$, Hamilton $\mathrm{G}$ et al (2018) Liver fat imaging-a clinical overview of ultrasound, CT, and MR imaging. Br J Radiol 91:20170959. https://doi.org/10.1259/bjr.20170959

53. El Kayal N, Lennartz S, Ekdawi S et al (2019) Value of spectral detector computed tomography for assessment of pancreatic lesions. Eur J Radiol 118:215-222. https://doi.org/10.1016/j.ejrad.2019.07.016

54. Nagayama $Y$, Tanoue S, Inoue T et al (2019) Dual-layer spectral CT improves image quality of multiphasic pancreas $\mathrm{CT}$ in patients with pancreatic ductal adenocarcinoma. Eur Radiol. https://doi.org/10.1007/ s00330-019-06337-y

55. Beer L, Toepker M, Ba-Ssalamah A et al (2019) Objective and subjective comparison of virtual monoenergetic vs. polychromatic images in patients with pancreatic ductal adenocarcinoma. Eur Radiol 29:3617-3625. https://doi.org/10.1007/s00330-019-06116-9

56. Noda Y, Goshima S, Kaga T et al (2019) Virtual monochromatic image at lower energy level for assessing pancreatic ductal adenocarcinoma in fast kV-switching dual-energy CT. Clin Radiol. https://doi.org/10.1016/j. crad.2019.11.012

57. Martin SS, Trapp F, Wichmann JL et al (2019) Dual-energy CT in early acute pancreatitis: improved detection using iodine quantification. Eur Radiol 29:2226-2232. https://doi.org/10.1007/s00330-018-5844-x

58. Mohammed MF, Elbanna KY, Mohammed AME et al (2018) Practical applications of dual-energy computed tomography in the acute abdomen. Radiol Clin North Am 56:549-563. https://doi.org/10.1016/j.rcl. 2018.03.004

59. Yin Q, Zou X, Zai X et al (2015) Pancreatic ductal adenocarcinoma and chronic mass-forming pancreatitis: Differentiation with dual-energy MDCT in spectral imaging mode. Eur J Radiol 84:2470-2476. https://doi. org/10.1016/j.ejrad.2015.09.023

60. Murray N, Darras KE, Walstra FE et al (2019) Dual-Energy CT in Evaluation of the Acute Abdomen. Radiographics 39:264-286. https://doi.org/ 10.1148/rg.2019180087

61. Li H, He D, Lao Q et al (2015) Clinical value of spectral CT in diagnosis of negative gallstones and common bile duct stones. Abdom Imaging 40:1587-1594. https://doi.org/10.1007/s00261-015-0387-y

62. Kiewiet JJS, Leeuwenburgh MMN, Bipat S et al (2012) A systematic review and meta-analysis of diagnostic performance of imaging in acute cholecystitis. Radiology 264:708-720. https://doi.org/10.1148/ radiol.12111561

63. Wertz JR, Lopez JM, Olson D, Thompson WM (2018) Comparing the diagnostic accuracy of ultrasound and $\mathrm{CT}$ in evaluating acute cholecystitis. AJR Am J Roentgenol 211:W92-W97. https://doi.org/10.2214/AJR. 17.18884

64. Morgan DE (2018) The role of dual-energy computed tomography in assessment of abdominal oncology and beyond. Radiol Clin North Am 56:565-585. https://doi.org/10.1016/j.rcl.2018.03.005

65. Ascenti G, Mileto A, Krauss B et al (2013) Distinguishing enhancing from nonenhancing renal masses with dual-source dual-energy CT: iodine quantification versus standard enhancement measurements. Eur Radiol 23:2288-2295. https://doi.org/10.1007/s00330-013-2811-4

66. Mileto A, Allen BC, Pietryga JA et al (2017) Characterization of incidental renal mass with dual-energy $C T$ : diagnostic accuracy of effective atomic number maps for discriminating nonenhancing cysts from enhancing masses. AJR Am J Roentgenol 209:W221-W230. https://doi.org/10. 2214/AJR.16.17325

67. Mileto A, Marin D, Ramirez-Giraldo JC et al (2014) Accuracy of contrastenhanced dual-energy MDCT for the assessment of iodine uptake in renal lesions. AJR Am J Roentgenol 202:W466-W474. https://doi.org/10 2214/AJR.13.11450

68. Bellini D, Panvini N, Laghi A et al (2019) Systematic review and metaanalysis investigating the diagnostic yield of dual-energy CT for renal mass assessment. AJR Am J Roentgenol. https://doi.org/10.2214/AJR. 18.20625

69. Mileto A, Sofue K, Marin D (2016) Imaging the renal lesion with dualenergy multidetector $\mathrm{CT}$ and multi-energy applications in clinical practice: what can it truly do for you? Eur Radiol 26:3677-3690. https:// doi.org/10.1007/s00330-015-4180-7 
70. Soesbe TC, Ananthakrishnan L, Lewis MA et al (2018) Pseudoenhancement effects on iodine quantification from dual-energy spectral CT systems: a multi-vendor phantom study regarding renal lesion characterization. Eur J Radiol 105:125-133. https://doi.org/10.1016/j.ejrad. 2018.06.002

71. Patel BN, Farjat A, Schabel C et al (2018) Energy-specific optimization of attenuation thresholds for low-energy virtual monoenergetic images in renal lesion evaluation. AJR Am J Roentgenol 210:W205-W217. https:// doi.org/10.2214/AJR.17.18641

72. Mileto A, Marin D, Alfaro-Cordoba M et al (2014) lodine quantification to distinguish clear cell from papillary renal cell carcinoma at dualenergy multidetector CT: a multireader diagnostic performance study. Radiology 273:813-820. https://doi.org/10.1148/radiol.14140171

73. Shuman WP, Mileto A, Busey JM et al (2019) Dual-energy CT urography With $50 \%$ reduced iodine dose versus single-energy CT urography with standard iodine dose. AJR Am J Roentgenol 212:117-123. https://doi. org/10.2214/AJR.18.19720

74. Ananthakrishnan L, Duan X, Xi Y et al (2018) Dual-layer spectral detector CT: non-inferiority assessment compared to dual-source dualenergy $\mathrm{CT}$ in discriminating uric acid from non-uric acid renal stones ex vivo. Abdom Radiol (NY) 43:3075-3081. https://doi.org/10.1007/ s00261-018-1589-x

75. Lombardo F, Bonatti M, Zamboni GA et al (2017) Uric acid versus nonuric acid renal stones: in vivo differentiation with spectral CT. Clin Radiol 72:490-496. https://doi.org/10.1016/j.crad.2017.01.018

76. Grosse Hokamp N, Salem J, Hesse A et al (2018) Low-dose characterization of kidney stones using spectral detector computed tomography: an ex vivo study. Invest Radiol 53:457-462. https://doi.org/10.1097/RLI. 0000000000000468

77. Adam SZ, Nikolaidis P, Horowitz JM et al (2016) Chemical shift MR imaging of the adrenal gland: principles, pitfalls, and applications. Radiographics 36:414-432. https://doi.org/10.1148/rg.2016150139

78. Helck A, Hummel N, Meinel FG et al (2014) Can single-phase dualenergy CT reliably identify adrenal adenomas? Eur Radiol 24:1636-1642. https://doi.org/10.1007/s00330-014-3192-z

79. Botsikas D, Triponez F, Boudabbous S et al (2014) Incidental adrenal lesions detected on enhanced abdominal dual-energy CT: can the diagnostic workup be shortened by the implementation of virtual unenhanced images? Eur J Radiol 83:1746-1751. https://doi.org/10. 1016/j.ejrad.2014.06.017

80. Connolly MJ, Mclnnes MDF, El-Khodary M et al (2017) Diagnostic accuracy of virtual non-contrast enhanced dual-energy CT for diagnosis of adrenal adenoma: a systematic review and meta-analysis. Eur Radiol 27:4324-4335. https://doi.org/10.1007/s00330-017-4785-0

81. Ju Y, Liu A, Dong Y et al (2015) The value of nonenhanced single-source dual-energy CT for differentiating metastases from adenoma in adrenal glands. Acad Radiol 22:834-839. https://doi.org/10.1016/j.acra.2015.03. 004

82. Liu W-D, Wu X-W, Hu J-M et al (2015) Monochromatic energy computed tomography image for active intestinal hemorrhage: a model investigation. World J Gastroenterol 21:214-220. https://doi.org/10.3748/wjg. V21.i1.214

83. Potretzke TA, Brace CL, Lubner MG et al (2015) Early small-bowel ischemia: dual-energy CT improves conspicuity compared with conventional CT in a swine model. Radiology 275:119-126. https://doi.org/ 10.1148/radiol.14140875

84. Lourenco PDM, Rawski R, Mohammed MF et al (2018) Dual-energy CT iodine mapping and 40-keV monoenergetic applications in the diagnosis of acute bowel ischemia. AJR Am J Roentgenol 211:564-570. https:// doi.org/10.2214/AJR.18.19554

85. Oda S, Nakaura T, Utsunomiya D et al (2017) Clinical potential of retrospective on-demand spectral analysis using dual-layer spectral detector-computed tomography in ischemia complicating smallbowel obstruction. Emerg Radiol 24:431-434. https://doi.org/10.1007/ s10140-017-1511-9

86. Darras KE, McLaughlin PD, Kang H et al (2016) Virtual monoenergetic reconstruction of contrast-enhanced dual energy CT at $70 \mathrm{keV}$ maximizes mural enhancement in acute small bowel obstruction. Eur J Radiol 85:950-956. https://doi.org/10.1016/j.ejrad.2016.02.019

87. Kim YS, Kim SH, Ryu HS, Han JK (2018) lodine quantification on spectral detector-based dual-energy CT enterography: correlation with
Crohn's disease activity index and external validation. Korean J Radiol 19:1077-1088. https://doi.org/10.3348/kjr.2018.19.6.1077

88. Peng JC, Feng Q, Zhu J et al (2016) Usefulness of spectral computed tomography for evaluation of intestinal activity and severity in ileocoIonic Crohn's disease. Therap Adv Gastroenterol 9:795-805. https://doi. org/10.1177/1756283X16668309

89. Lee SM, Kim SH, Ahn SJ et al (2018) Virtual monoenergetic dual-layer, dual-energy CT enterography: optimization of keV settings and its added value for Crohn's disease. Eur Radiol 28:2525-2534. https://doi. org/10.1007/s00330-017-5215-Z

90. Elbanna KY, Mohammed MF, Chahal T et al (2018) Dual-energy CT in differentiating nonperforated gangrenous appendicitis from uncomplicated appendicitis. AJR Am J Roentgenol 211:776-782. https://doi.org/ 10.2214/AJR.17.19274

91. Eliahou R, Azraq Y, Carmi R et al (2010) Dual-energy based spectral electronic cleansing in non-cathartic computed tomography colonography: an emerging novel technique. Semin Ultrasound CT MR 31:309-314. https://doi.org/10.1053/j.sult.2010.05.005

92. Taguchi N, Oda S, Imuta M et al (2018) Dual-energy computed tomography colonography using dual-layer spectral detector computed tomography: Utility of virtual monochromatic imaging for electronic cleansing. Eur J Radiol 108:7-12. https://doi.org/10.1016/j.ejrad.2018.09. 011

93. Tachibana R, Näppi JJ, Ota J, et al (2018) Deep Learning Electronic Cleansing for Single- and Dual-Energy CT Colonography. Radiographics 38:2034-2050. https://doi.org/10.1148/rg.2018170173

94. Yeh BM, Obmann MM, Westphalen AC et al (2018) Dual energy computed tomography scans of the bowel: benefits, pitfalls, and future directions. Radiol Clin North Am 56:805-819. https://doi.org/10.1016/j. rcl.2018.05.002

95. Wei W, Yu Y, Lv W et al (2014) Predictive value of dual-energy spectral computed tomographic imaging on the histological origin of carcinomas in the ampullary region. Abdom Imaging 39:702-710. https://doi. org/10.1007/s00261-014-0098-9

96. Yang C-B, Yu N, Jian Y-J et al (2019) Spectral CT imaging in the differential diagnosis of small bowel adenocarcinoma from primary small intestinal lymphoma. Acad Radiol 26:878-884. https://doi.org/10.1016/j. acra.2018.08.020

97. Zhou Y, Hou P, Zha Ket al (2019) Spectral computed tomography for the quantitative assessment of patients with carcinoma of the gastroesophageal junction: initial differentiation between a diagnosis of squamous cell carcinoma and adenocarcinoma. J Comput Assist Tomogr 43:187-193. https://doi.org/10.1097/RCT.0000000000000826

98. Zhang X, Bai L, Wang D et al (2019) Gastrointestinal stromal tumor risk classification: spectral CT quantitative parameters. Abdom Radiol (NY) 44:2329-2336. https://doi.org/10.1007/s00261-019-01973-w

99. Gong H-X, Zhang K-B, Wu L-M et al (2016) Dual energy spectral CT imaging for colorectal cancer grading: a preliminary study. PLoS One 11:e0147756. https://doi.org/10.1371/journal.pone.0147756

100. Yang Z, Zhang $X$, Fang $M$ et al (2019) Preoperative diagnosis of regional lymph node metastasis of colorectal cancer with quantitative parameters from dual-energy CT. AJR Am J Roentgenol. https://doi.org/10. 2214/AJR.18.20843

101. Al-Najami I, Lahaye MJ, Beets-Tan RGH, Baatrup G (2017) Dual-energy CT can detect malignant lymph nodes in rectal cancer. Eur J Radiol 90:81-88. https://doi.org/10.1016/j.ejrad.2017.02.005

102. Zhou Z, Liu Y, Meng K et al (2019) Application of spectral CT imaging in evaluating lymph node metastasis in patients with gastric cancers: initial findings. Acta Radiol 60:415-424. https://doi.org/10.1177/02841 85118786076

103. Al-Najami I, Drue HC, Steele R, Baatrup G (2017) Dual energy CT-a possible new method to assess regression of rectal cancers after neoadjuvant treatment. J Surg Oncol 116:984-988. https://doi.org/10.1002/ jso.24761

104. Benveniste AP, de Castro FS, Broering G et al (2017) Potential application of dual-energy $C T$ in gynecologic cancer: initial experience. AJR Am J Roentgenol 208:695-705. https://doi.org/10.2214/AJR.16.16227

105. Elsherif SB, Zheng S, Ganeshan D et al (2020) Does dual-energy CT differentiate benign and malignant ovarian tumours? Clin Radiol 75:606-614. https://doi.org/10.1016/j.crad.2020.03.006 
106. Rizzo S, Femia M, Radice D et al (2018) Evaluation of deep myometrial invasion in endometrial cancer patients: is dual-energy CT an option? Radiol Med 123:13-19. https://doi.org/10.1007/s11547-017-0810-2

107. Jiang C, Yang P, Lei J et al (2017) The application of iodine quantitative information obtained by dual-source dual-energy computed tomography on chemoradiotherapy effect monitoring for cervical cancer: a preliminary study. J Comput Assist Tomogr 41:737-745. https://doi.org/ 10.1097/RCT.0000000000000603

108. Tawfik AM, Razek AA, Kerl JM et al (2014) Comparison of dual-energy CT-derived iodine content and iodine overlay of normal, inflammatory and metastatic squamous cell carcinoma cervical lymph nodes. Eur Radiol 24:574-580. https://doi.org/10.1007/s00330-013-3035-3

\section{Publisher's Note}

Springer Nature remains neutral with regard to jurisdictional claims in published maps and institutional affiliations.

\section{Submit your manuscript to a SpringerOpen ${ }^{\circ}$ journal and benefit from:}

- Convenient online submission

- Rigorous peer review

- Open access: articles freely available online

- High visibility within the field

- Retaining the copyright to your article

Submit your next manuscript at springeropen.com 